\title{
NEW STRIPS OF CONVERGENCE FOR DIRICHLET SERIES
}

\author{
Andreas Defant, Domingo García, and Manuel Maestre
}

\begin{abstract}
In this article we study the interplay of the theory of classical Dirichlet series in one complex variable with recent development on monomial expansions of holomorphic functions in infinitely many variables. For a given Dirichlet series we obtain new strips of convergence in the complex plane related to Bohr's classical strips of uniform but non absolute convergence.
\end{abstract}

\section{Introduction}

Harald Bohr in [7] studied the width of the strip of uniform but non absolute convergence of a Dirichlet series $\sum_{n=1}^{\infty} \frac{a_{n}}{n^{s}}$ on $\mathbb{C}$. He made a deep connection with infinite dimensional holomorphy. His work is very much based on the fact that if $p=\left(p_{k}\right)_{k=1}^{\infty}$ is the sequence of prime numbers, then the sequence $\left(\log p_{k}\right)_{k=1}^{\infty}$ is linearly independent over the field $\mathbb{Q}$. Actually he associated to each series $\sum_{n=1}^{\infty} \frac{a_{n}}{n^{s}}$ a family of formal power series $\sum_{\alpha \in \mathbb{N}_{0}^{(\mathbb{N})}} \frac{a_{p^{\alpha}}}{p^{\alpha \sigma}} z^{\alpha}$ in infinitely many variables on the infinite dimensional polydisk $\mathbb{D}^{\mathbb{N}}$, where $\sigma \in \mathbb{R}$ and $p^{\alpha}=p_{1}^{\alpha_{1}} \ldots p_{k}^{\alpha_{k}}$ for $\alpha=\left(\alpha_{1}, \ldots, \alpha_{k}, 0, \ldots\right) \in \mathbb{N}_{0}^{(\mathbb{N})}$. For a fixed Dirichlet series $\sum_{n=1}^{\infty} \frac{a_{n}}{n^{s}}$ he defined the maximal half plane $\left[\operatorname{Re}>\sigma_{a}\right]$ of absolute convergence where the so-called abscissa of absolute convergence is given by

$$
\begin{aligned}
\sigma_{a}=\inf \left\{r \in \mathbb{R}: \sum_{n=1}^{\infty} \frac{a_{n}}{n^{s}}\right. \text { is absolutely convergent } \\
\text { on the half plane }[\operatorname{Re} s \geq r]\},
\end{aligned}
$$

2000 Mathematics Subject Classification. Primary: 30B50; Secondary: 32A05.

Key words. Dirichlet series, power series, polynomials.

The authors were supported by MICINN and FEDER Proyect MTM2008-03211. The third author was also supported by Prometeo 2008/101. 
as well as the maximal half plane $\left[\operatorname{Re}>\sigma_{u}\right]$ of uniform convergence with the abscissa of uniform convergence given by

$$
\begin{aligned}
\sigma_{u}=\inf \left\{r \in \mathbb{R}: \sum_{n=1}^{\infty} \frac{a_{n}}{n^{s}}\right. \text { is uniformly convergent } \\
\text { on the half plane }[\operatorname{Re} s \geq r]\} .
\end{aligned}
$$

Moreover, Bohr introduced the notation

$$
T=\sup \left\{\sigma_{a}-\sigma_{u}: \sum_{n=1}^{\infty} \frac{a_{n}}{n^{s}}\right\},
$$

the maximal width where a Dirichlet series $\sum_{n=1}^{\infty} \frac{a_{n}}{n^{s}}$ converges uniformly but not absolutely. Bohr's main aim then was to find the precise value of $T$. He himself was only able to prove that $T \leq \frac{1}{2}$, whereas the final result $T=\frac{1}{2}$ was obtained in 1931 by Bohnenblust and Hille in [5]. But Bohr in [7] contributed with two crucial facts to this solution. The first one is that $\sigma_{u}=h$ where

(1.2) $h=\inf \left\{\sigma \in \mathbb{R}: \sum_{\alpha \in \mathbb{N}_{0}^{m} \times\{0\}}\left|\frac{a_{p^{\alpha}}}{p^{\sigma \alpha}} z^{\alpha}\right|<\infty\right.$ for all $z \in B_{\ell_{\infty}^{m}}, \quad m=1,2, \ldots$

$$
\text { and } \left.\sup _{m \in \mathbb{N}} \sup _{z \in B_{\ell_{\infty}^{m}}}\left|\sum_{\alpha \in \mathbb{N}_{0}^{m} \times\{0\}} \frac{a_{p^{\alpha}}}{p^{\sigma \alpha}} z^{\alpha}\right|<\infty\right\}
$$

(here as usual $B_{\ell_{\infty}^{m}}:=\mathbb{C}^{m} \times\{0\} \cap B_{\ell_{\infty}}$ ). The other one is that

$$
T=\frac{1}{S}
$$

where

$$
\begin{aligned}
& S=\sup \left\{q \geq 1: \sum_{\alpha \in \mathbb{N}_{0}^{(N)}}\left|c_{\alpha} u^{\alpha}\right|<\infty \quad \text { for all } u \in \ell_{q} \cap B_{\ell_{\infty}},\right. \\
& \text { for all power series } \sum_{\alpha \in \mathbb{N}_{0}^{(\mathbb{N})}} c_{\alpha} z^{\alpha} \text { such that } \sum_{\alpha \in \mathbb{N}_{0}^{m} \times\{0\}}\left|c_{\alpha} z^{\alpha}\right|<\infty \\
& \text { for all } z \in B_{\ell_{\infty}^{m}}, \quad m=1,2, \ldots \\
& \text { and } \left.\sup _{m \in \mathbb{N}} \sup _{z \in B_{\ell \infty}^{m}}\left|\sum_{\alpha \in \mathbb{N}_{0}^{m} \times\{0\}} c_{\alpha} z^{\alpha}\right|<\infty\right\} \text {. }
\end{aligned}
$$


With this notation $T=1 / S=1 / 2$, a result which we call the BohrBohnenblust-Hille Theorem.

In order to reformulate Bohr's definitions of $T$ and $S$ in terms of (infinite dimensional) complex analysis we need some more definitions. A (non empty) open subset $R$ of $\ell_{w}, 1 \leq w \leq \infty$, is called a (complete) Reinhardt domain if given $x=\left(x_{n}\right) \in R$, then $y=\left(y_{n}\right) \in R$ for every $y \in \mathbb{C}^{\mathbb{N}}$ for which $\left|y_{n}\right| \leq\left|x_{n}\right|$ for all $n$. A function $f: R \longrightarrow \mathbb{C}$ is called Gâteaux holomorphic if its restriction to the intersection with any finite dimensional subspace of $\ell_{w}$ is holomorphic as a function of several complex variables. Finally, $f$ is holomorphic if it is Fréchet differentiable on $R$ (see $[\mathbf{1 4}],[\mathbf{1 6}]$ for background and properties used through the paper). Given a Gâteaux holomorphic function $f: R \longrightarrow \mathbb{C}$, its monomial expansion is the formal power series $\sum_{\alpha \in \mathbb{N}_{0}^{(\mathbb{N})}} c_{\alpha} z^{\alpha}$ where

$$
c_{\alpha}(f)=\left(\frac{1}{2 \pi i}\right)^{n} \int_{\left|z_{1}\right|=r_{1}} \ldots \int_{\left|z_{n}\right|=r_{n}} \frac{f\left(\sum_{j=1}^{n} z_{j} e_{j}\right)}{z^{\alpha+1}} d z_{1} \ldots d z_{n}
$$

for every $n$ such that $\alpha_{m}=0$ for $m>n$ and any $r_{1}, \ldots, r_{n}$ satisfying $\left[\left|z_{1}\right| \leq r_{1}\right] \times \cdots \times\left[\left|z_{n}\right| \leq r_{n}\right] \times\{0\} \subset R$ (i.e. the coefficients obtained when $f$ is considered as a function just of the first $n$ complex variables). Given a set $\mathcal{F}(R)$ of holomorphic functions on a Reinhardt domain $R$ of $\ell_{w}$ we call the set

$$
\operatorname{dom} \mathcal{F}(R)=\left\{z \in R: \sum_{\alpha \in \mathbb{N}_{0}^{(\mathbb{N})}}\left|c_{\alpha}(f) z^{\alpha}\right|<\infty, \text { for all } f \in \mathcal{F}(R)\right\}
$$

domain of convergence of $\mathcal{F}(R)$.

It is nowadays well known (see Lemma 2.2) that a formal power series $\sum_{\alpha \in \mathbb{N}_{0}^{(\mathbb{N})}} c_{\alpha} z^{\alpha}$ satisfies $\sup _{n \in \mathbb{N}} \sup _{\|z\|_{\infty} \leq 1}\left|\sum_{\alpha \in \mathbb{N}_{0}^{n} \times\{0\}} c_{\alpha} z^{\alpha}\right|<\infty$ if and only if there exists a function $f \in H_{\infty}\left(B_{\ell_{\infty}}\right)$ such that $c_{\alpha}(f)=c_{\alpha}$ for every $\alpha \in \mathbb{N}_{0}^{(\mathbb{N})}$. When there is no risk of confusion we will denote by $\sum_{\alpha \in \mathbb{N}_{0}^{(\mathbb{N})}} c_{\alpha} z^{\alpha}$ both a formal power series and its associated function $f$ (whenever it exists).

With this more modern point of view we obtain for every Dirichlet series $\sum_{n=1}^{\infty} \frac{a_{n}}{n^{s}}$ the following two alternative descriptions of $T$ and $S$ :

$$
\sigma_{u}=h=\inf \left\{\sigma \in \mathbb{R}: g_{\sigma}(z)=\sum_{\alpha \in \mathbb{N}_{0}^{(\mathbb{N})}} \frac{a_{p^{\alpha}}}{p^{\alpha \sigma}} z^{\alpha} \in H_{\infty}\left(B_{\ell_{\infty}}\right)\right\},
$$


and

$$
S=\sup \left\{q \geq 1: \ell_{q} \cap B_{\ell_{\infty}} \subset \operatorname{dom} H_{\infty}\left(B_{\ell_{\infty}}\right)\right\} .
$$

Very recently a thorough study on domains of convergence of monomial expansions of holomorphic functions has been made in [13]; it contains the following result that will be a key ingredient to us.

Theorem 1.1 ([13, Example 4.9]). Let $R$ be a Reinhardt domain in $\ell_{w}$ with $1 \leq w \leq \infty, \mathcal{F}(R)$ a set of holomorphic functions on $R$ which contains the polynomials, and $\varepsilon>0$. Then for $1 \leq w<2$ we have

$$
\ell_{1} \cap R \subset \operatorname{dom} \mathcal{F}(R) \subset \ell_{1+\varepsilon} \cap R,
$$

and for $w \geq 2$ with $\frac{1}{q}=\frac{1}{2}+\frac{1}{w}$

$$
\ell_{q} \cap R \subset \operatorname{dom} \mathcal{F}(R) \subset \ell_{q+\varepsilon} \cap R .
$$

In particular, by taking $w=\infty$ the above result implies that $S=2$ and we recover the Bohr-Bohnenblust-Hille Theorem.

The aim of this paper is to clarify what kind of new information on Dirichlet series can be obtained from the new results on domains of convergence of monomials expansions on Reinhardt domains of $\ell_{w}$, $1 \leq w<\infty$, in particular from Theorem 1.1. We will focus on new strips of convergence for Dirichlet series closely related to the one studied by Bohr in [7] and Bohnenblust and Hille in [5]: Consider in $\ell_{w}$ the Reinhardt domain

$$
\ell_{w} \cap \mathbb{D}^{\mathbb{N}}
$$

where $\mathbb{D}$ as usual denotes the open unit disk in $\mathbb{C}$ and fix a set $\mathcal{F}\left(\ell_{w} \cap \mathbb{D}^{\mathbb{N}}\right)$ of holomorphic functions with a technical property $(\mathrm{T})$ (see the definition below); concrete examples are the space $H\left(\ell_{w} \cap \mathbb{D}^{\mathbb{N}}\right)$ of all holomorphic functions, or the space $H_{\beta}\left(\ell_{w} \cap \mathbb{D}^{\mathbb{N}}\right)$ of all holomorphic functions that are bounded on bounded subsets of $\ell_{w} \cap \mathbb{D}^{\mathbb{N}}$, or the space $H_{b}\left(\ell_{w} \cap \mathbb{D}^{\mathbb{N}}\right)$ of all holomorphic functions of bounded type (see [14, p. 164] for the definition and properties).

For every such function space $\mathcal{F}\left(\ell_{w} \cap \mathbb{D}^{\mathbb{N}}\right)$ we will associate to each Dirichlet series $\sum_{n=1}^{\infty} \frac{a_{n}}{n^{s}}$ a new line $h\left(\mathcal{F}\left(\ell_{w} \cap \mathbb{D}^{\mathbb{N}}\right)\right)$ of (monomial) convergence (see the definition in Section 2 below).

This new line in the case $\mathcal{F}\left(\ell_{w} \cap \mathbb{D}^{\mathbb{N}}\right)=H_{\infty}\left(\ell_{w} \cap \mathbb{D}^{\mathbb{N}}\right), 1 \leq w \leq \infty$, coincides with the old line $\sigma_{u}$ (see Proposition 2.3). For other function spaces $\mathcal{F}\left(\ell_{w} \cap \mathbb{D}^{\mathbb{N}}\right)$ different from $H_{\infty}\left(\ell_{w} \cap \mathbb{D}^{\mathbb{N}}\right)$ the situation is very different. In Theorem 2.5 we prove the inequality $\sigma_{u} \leq h\left(\mathcal{F}\left(\ell_{w} \cap \mathbb{D}^{\mathbb{N}}\right)\right) \leq$ $\sigma_{u}+\frac{1}{w}$ (provided $\mathcal{F}\left(\ell_{w} \cap \mathbb{D}^{\mathbb{N}}\right)$ satisfies property $\left.(\mathrm{T})\right)$. Moreover, we extend (in a straightforward way) Bohr's definitions of $S$ and $T$ to the 
families $\mathcal{F}\left(\ell_{w} \cap \mathbb{D}^{\mathbb{N}}\right)$ (for the definitions see Section 3). Then in Proposition 3.1 we recover and generalize the original Bohr-Bohnenblust-Hille Theorem: For each $1 \leq w \leq \infty$

$$
T\left(H_{\infty}\left(\ell_{w} \cap \mathbb{D}^{\mathbb{N}}\right)\right)=\frac{1}{S\left(H_{\infty}\left(\ell_{w} \cap \mathbb{D}^{\mathbb{N}}\right)\right)}=\frac{1}{2} .
$$

Again the situation changes for general function spaces $\mathcal{F}\left(\ell_{w} \cap \mathbb{D}^{\mathbb{N}}\right.$ ) (satisfying $(\mathrm{T})$ ): in Theorem 3.2 we show the formulas

$$
T\left(\mathcal{F}\left(\ell_{w} \cap \mathbb{D}^{\mathbb{N}}\right)\right)=\frac{1}{S\left(\mathcal{F}\left(\ell_{w} \cap \mathbb{D}^{\mathbb{N}}\right)\right)}= \begin{cases}1 & \text { if } 1 \leq w<2 \\ \frac{1}{2}+\frac{1}{w} & \text { if } 2 \leq w \leq \infty\end{cases}
$$

\section{New abscissas of convergence}

Fix some Reinhardt domain $R$ in $\ell_{w}, 1 \leq w \leq \infty$, and a set $\mathcal{F}(R)$ of holomorphic functions on $R$ and a Dirichlet series $\sum_{n=1}^{\infty} \frac{a_{n}}{n^{s}}$. We define

$$
h(\mathcal{F}(R)):=\inf \left\{\sigma \in \mathbb{R}: g_{\sigma}(z)=\sum_{\alpha \in \mathbb{N}_{0}^{(\mathbb{N})}} \frac{a_{p^{\alpha}}}{p^{\alpha \sigma}} z^{\alpha} \in \mathcal{F}(R)\right\} .
$$

Consider $\mathcal{F}(R)$ a set of holomorphic functions remaining stable under composition with diagonal operators of norm $\leq 1$ (i.e. given $b=$ $\left(b_{n}\right) \subset \mathbb{C}^{\mathbb{N}}$ with $\left|b_{n}\right| \leq 1$ for all $n$, and $f \in \mathcal{F}(R)$ then $g \in \mathcal{F}(R)$ where $g(z)=f(b \cdot z), z \in R)$. In that case $h(\mathcal{F}(R))$ defines a half plane of $\mathbb{C}$ in the following sense: if $s \in \mathbb{C}$ and $\operatorname{Re} s>h(\mathcal{F}(R))$ then $g_{s}(z)=\sum_{\alpha \in \mathbb{N}_{0}^{(\mathbb{N})}} \frac{a_{p^{\alpha}}}{p^{\alpha s}} z^{\alpha} \in \mathcal{F}(R)$; indeed, let Re $s>\sigma \geq h(\mathcal{F}(R))$ be such that $g_{\sigma}(z)=\sum_{\alpha \in \mathbb{N}_{0}^{(\mathbb{N})}} \frac{a_{p^{\alpha}}}{p^{\alpha \sigma}} z^{\alpha} \in \mathcal{F}(R)$. Then

$$
g_{s}(z)=\sum_{\alpha \in \mathbb{N}_{0}^{(\mathbb{N})}} \frac{a_{p^{\alpha}}}{p^{\alpha \sigma}}\left(\frac{z}{p^{s-\sigma}}\right)^{\alpha}=g_{\sigma}\left(\frac{z}{p^{s-\sigma}}\right) .
$$

Given $1 \leq w \leq \infty$, we take the canonical inclusion $i_{w}: \ell_{w} \longrightarrow \ell_{\infty}$ and we denote by $\ell_{w} \cap \mathbb{D}^{\mathbb{N}}$ the open subset $i_{w}^{-1}\left(B_{\ell_{\infty}}\right)$ of $\ell_{w}$. Clearly $\ell_{w} \cap \mathbb{D}^{\mathbb{N}}$ is a Reinhardt domain in $\ell_{w}$. We are going to work with sets of holomorphic functions defined on these Reinhardt domains, since, after a careful analysis of the original proofs, it seems to us that they are the only ones which allow to translate Bohr's study from $\ell_{\infty}$ to $\ell_{w}$.

The first result shows that the abscissa $h\left(\mathcal{F}\left(\ell_{w} \cap \mathbb{D}^{\mathbb{N}}\right)\right)$ is less than or equal to the absolute convergence abscissa $\sigma_{a}$, and the maximal width between both is $\leq 1$. 
Proposition 2.1. Let $\mathcal{F}\left(\ell_{w} \cap \mathbb{D}^{\mathbb{N}}\right)$, for $1 \leq w \leq \infty$, be a set of holomorphic functions remaining stable under composition with diagonal operators of norm $\leq 1$ and containing the bounded holomorphic functions on $\ell_{w} \cap \mathbb{D}^{\mathbb{N}}$. Given a Dirichlet series, $\sum_{n=1}^{\infty} \frac{a_{n}}{n^{s}}$, then $h\left(\mathcal{F}\left(\ell_{w} \cap \mathbb{D}^{\mathbb{N}}\right)\right)$ is $f_{-}$nite if and only if $\sigma_{a}$ is finite too and, in that case, $h\left(\mathcal{F}\left(\ell_{w} \cap \mathbb{D}^{\mathbb{N}}\right)\right) \leq \sigma_{a} \leq$ $h\left(\mathcal{F}\left(\ell_{w} \cap \mathbb{D}^{\mathbb{N}}\right)\right)+1$. Moreover, $\sigma_{a}=+\infty$ if and only if $h\left(\mathcal{F}\left(\ell_{w} \cap \mathbb{D}^{\mathbb{N}}\right)\right)=$ $+\infty$ and $\sigma_{a}=-\infty$ if and only if $h\left(\mathcal{F}\left(\ell_{w} \cap \mathbb{D}^{\mathbb{N}}\right)\right)=-\infty$.

Proof: First we check that if there exists $s \in \mathbb{C}$ such that $\sum_{\alpha \in \mathbb{N}_{0}^{(\mathbb{N})}}\left|\frac{a_{p^{\alpha}}}{p^{\alpha s}}\right|<$ $\infty$, then $h\left(\mathcal{F}\left(\ell_{w} \cap \mathbb{D}^{\mathbb{N}}\right)\right) \leq \operatorname{Re} s$. Since

$$
\left|\frac{a_{p^{\alpha}}}{p^{\alpha \operatorname{Re} s}}\right||z|^{\alpha} \leq\left|\frac{a_{p^{\alpha}}}{p^{\alpha \operatorname{Re} s}}\right|
$$

for all $z \in \ell_{w} \cap \mathbb{D}^{\mathbb{N}}$, and $\sum_{\alpha \in \mathbb{N}_{0}^{(\mathbb{N})}}\left|\frac{a_{p^{\alpha}}}{p^{\alpha \operatorname{Re} s}}\right|<\infty$, we see that $g_{\operatorname{Re} s}(z)=$ $\sum_{\alpha \in \mathbb{N}_{0}^{(\mathbb{N})}} \frac{a_{p^{\alpha}}}{p^{\alpha e_{s}}} z^{\alpha}$ is a bounded holomorphic function on $\ell_{w} \cap \mathbb{D}^{\mathbb{N}}$, thus $g_{\operatorname{Re} s} \in \mathcal{F}\left(\ell_{w} \cap \mathbb{D}^{\mathbb{N}}\right)$ and $h\left(\mathcal{F}\left(\ell_{w} \cap \mathbb{D}^{\mathbb{N}}\right)\right) \leq \operatorname{Re} s$.

To finish the proof it is enough to see that there exists $s \in \mathbb{C}$ for which $g_{s}(z)=\sum_{\alpha \in \mathbb{N}_{0}^{(N)}} \frac{a_{p^{\alpha}}}{p^{\alpha s}} z^{\alpha} \in \mathcal{F}\left(\ell_{w} \cap \mathbb{D}^{\mathbb{N}}\right)$, then $\sigma_{a} \leq \operatorname{Re} s+1$. Let $\sigma>\operatorname{Re} s+1$. We know from Theorem 1.1 (see also [13, Theorem 3.6]) that $\ell_{1} \cap \ell_{w} \cap \mathbb{D}^{\mathbb{N}} \subset \operatorname{dom} \mathcal{F}\left(\ell_{w} \cap \mathbb{D}^{\mathbb{N}}\right)$. But $\sigma-\operatorname{Re} s>1$ implies that $\left(\frac{1}{p^{\sigma-R e s}}\right) \in \ell_{1} \cap \mathbb{D}^{\mathbb{N}} \subset \ell_{w} \cap \mathbb{D}^{\mathbb{N}}$. Thus

$$
\sum_{\alpha \in \mathbb{N}_{0}^{(\mathbb{N})}}\left|\frac{a_{p^{\alpha}}}{p^{\alpha \sigma}}\right|=\sum_{\alpha \in \mathbb{N}_{0}^{(\mathbb{N})}}\left|\frac{a_{p^{\alpha}}}{p^{s}}\right|\left|\left(\frac{1}{p^{\sigma-\operatorname{Re} s}}\right)^{\alpha}\right|<\infty,
$$

the conclusion.

Now we are going to show that $h\left(H_{\infty}\left(\ell_{w} \cap \mathbb{D}^{\mathbb{N}}\right)\right)$ for each Dirichlet series and each possible $w$ is nothing else than the original abscissa of uniform convergence. The following lemma is needed. It should be well known and is just proved for the sake of completeness.

Lemma 2.2. Let $\sum_{\alpha \in \mathbb{N}_{0}^{(\mathbb{N})}} c_{\alpha} z^{\alpha}$ be a formal power series such that, for every $m \in \mathbb{N}, \sum_{\alpha \in \mathbb{N}_{0}^{m} \times\{0\}} c_{\alpha} z^{\alpha}$ is absolutely convergent at any point of $\mathbb{C}^{(\mathbb{N})} \cap B_{\ell_{\infty}}$ and that

$$
\sup _{m \in \mathbb{N}} \sup _{z \in B_{\ell m}^{m}}\left|\sum_{\alpha \in \mathbb{N}_{0}^{m} \times\{0\}} c_{\alpha} z^{\alpha}\right|<\infty .
$$

Then there exists $f \in H_{\infty}\left(B_{\ell_{\infty}}\right)$ such that $c_{\alpha}(f)=c_{\alpha}$, for every $\alpha \in \mathbb{N}_{0}^{(\mathbb{N})}$. 
Proof: Consider the normed space $Z:=\left(\mathbb{C}^{(\mathbb{N})},\|\cdot\|_{\infty}\right)$. Clearly $g(z)=$ $\sum_{\alpha \in \mathbb{N}_{0}^{m} \times\{0\}} c_{\alpha} z^{\alpha}, z \in B_{Z}$, defines a Gâteaux holomorphic function on $B_{Z}$ that, moreover, is bounded and hence holomorphic on $B_{Z}([\mathbf{1 4}$, Proposition 3.7]). Thus there exists a sequence $\left(P_{m}\right)$ of continuous $m$-homogeneous polynomials on $Z$ with

$$
g(z)=\sum_{m=0}^{\infty} P_{m}(z)
$$

for all $z \in B_{Z}$. If we fix $0<r<1$ then, by the Cauchy inequalities,

$$
\frac{1}{r^{m}}\left\|P_{m}\right\|_{r B_{Z}} \leq\|g\|_{B_{Z}}<\infty .
$$

That implies that the Taylor series of $g$ at zero converges absolutely and uniformly on $r B_{Z}$. Thus $g$ is uniformly continuous on $r B_{Z}$. As a consequence there exists a unique uniformly continuous extension $\widetilde{g}$ to $r \bar{B}_{Z}=r \bar{B}_{c_{0}}$. Analogously, each $P_{m}$ has a unique uniformly continuous extension to $c_{0}$ which is an $m$-homogeneous polynomial and will be denoted by $\widetilde{P_{m}}$. Moreover, by $(2.1)$

$$
\left\|\widetilde{P_{m}}\right\|_{r B_{c_{0}}}=\left\|P_{m}\right\|_{r B_{Z}} \leq r^{m}\|g\|_{B_{Z}},
$$

for all $m$ and all $0<r<1$. Hence the series $\sum_{m=0}^{\infty} \widetilde{P_{m}}$ converges absolutely and uniformly on $r B_{c_{0}}, 0<r<1$, defining a holomorphic function on $B_{c_{0}}$. Since it is uniformly continuous on $r B_{c_{0}}, 0<r<1$, we conclude that it coincides with $\widetilde{g}$, i.e. $\widetilde{g} \in H_{\infty}\left(B_{c_{0}}\right)$. Now, by the classical result by Aron and Berner from [1] (in the Davie-Gamelin version [9, Theorem 5]), $\widetilde{g}$ has an extension to another bounded holomorphic function $f$ on $B_{\ell_{\infty}}$. Clearly,

for every $\alpha \in \mathbb{N}_{0}^{(\mathbb{N})}$.

$$
c_{\alpha}(f)=c_{\alpha}(g)=c_{\alpha},
$$

The following result extends Bohr's description of the uniform convergence abscissa $\sigma_{u}$ from (1.2) and (1.4).

Proposition 2.3. For each Dirichlet series $\sum_{n=1}^{\infty} \frac{a_{n}}{n^{s}}$ we have

$$
h\left(H_{\infty}\left(\ell_{w} \cap \mathbb{D}^{\mathbb{N}}\right)\right)=h\left(H_{\infty}\left(B_{\ell_{\infty}}\right)\right)=\sigma_{u},
$$

for every $1 \leq w \leq \infty$.

Proof: We check that $h\left(H_{\infty}\left(B_{\ell_{\infty}}\right)\right) \leq h\left(H_{\infty}\left(\ell_{w} \cap \mathbb{D}^{\mathbb{N}}\right)\right)$. Given $\sigma>$ $h\left(H_{\infty}\left(\ell_{w} \cap \mathbb{D}^{\mathbb{N}}\right)\right)$, there exists a holomorphic and bounded function $g_{\sigma}$ : $\ell_{w} \cap \mathbb{D}^{\mathbb{N}} \longrightarrow \mathbb{C}$ with monomial expansion $\sum_{\alpha \in \mathbb{N}_{0}^{(\mathbb{N})}} \frac{a_{p} \alpha}{p^{\alpha \sigma}} z^{\alpha}$, i.e. $c_{\alpha}\left(g_{\sigma}\right)=$ $\frac{a_{p^{\alpha}}}{p^{\alpha \sigma}}$ for every $\alpha \in \mathbb{N}_{0}^{(\mathbb{N})}$. Consider the normed space $Z:=\left(\mathbb{C}^{(\mathbb{N})},\|\cdot\|_{\infty}\right)$. 
Since $B_{Z}$, the open unit ball of $Z$, is included in $\ell_{w} \cap \mathbb{D}^{\mathbb{N}}$ and all norms on finite dimensional spaces are equivalent, the restriction of $g_{\sigma}$ to $B_{Z}$ is Gâteaux holomorphic and bounded. By the above lemma, there exists a bounded holomorphic function $f_{\sigma} \in H_{\infty}\left(B_{\ell_{\infty}}\right)$ which is an extension of $g_{\sigma}$ and satisfies $c_{\alpha}\left(f_{\sigma}\right)=\frac{a_{p^{\alpha}}}{p^{\alpha \sigma}}$ for every $\alpha \in \mathbb{N}_{0}^{(\mathbb{N})}$. Hence $h\left(H_{\infty}\left(B_{\ell_{\infty}}\right)\right) \leq \sigma$.

The other inequality is straightforward. Consider $\sigma>h\left(H_{\infty}\left(B_{\ell_{\infty}}\right)\right)$, there exists a function $s_{\sigma}: \ell_{w} \cap \mathbb{D}^{\mathbb{N}} \longrightarrow \mathbb{C}$ holomorphic and bounded on $B_{\ell_{\infty}}$ with monomial expansion $\sum_{\alpha \in \mathbb{N}_{0}^{(\mathbb{N})}} \frac{a_{p^{\alpha}}}{p^{\alpha \sigma}} z^{\alpha}$, i.e. $c_{\alpha}\left(s_{\sigma}\right)=\frac{a_{p} \alpha}{p^{\alpha \sigma}}$ for every $\alpha \in \mathbb{N}_{0}^{(\mathbb{N})}$. Using again the equivalence of all the norms on finite dimensional spaces, the restriction $s_{\sigma \mid}$ of $s_{\sigma}$ to $\ell_{w} \cap \mathbb{D}^{\mathbb{N}}$ is Gâteaux holomorphic and bounded on $\ell_{w} \cap \mathbb{D}^{\mathbb{N}}$, thus it is holomorphic (with the $\|\cdot\|_{w}$ norm) on $\ell_{w} \cap \mathbb{D}^{\mathbb{N}}$. As the monomial expansion of $s_{\sigma}$ and $s_{\sigma \mid}$ coincide we get $h\left(H_{\infty}\left(\ell_{w} \cap \mathbb{D}^{\mathbb{N}}\right)\right) \leq \sigma$ which implies $h\left(H_{\infty}\left(\ell_{w} \cap \mathbb{D}^{\mathbb{N}}\right)\right) \leq$ $h\left(H_{\infty}\left(B_{\ell_{\infty}}\right)\right)$.

Motivated by the preceding result the next aim is to link for a given Dirichlet series $\sum_{n=1}^{\infty} \frac{a_{n}}{n^{s}}$ and a wide class of function spaces $\mathcal{F}\left(\ell_{w} \cap\right.$ $\left.\mathbb{D}^{\mathbb{N}}\right)$ the (new) abscissa $h\left(\mathcal{F}\left(\ell_{w} \cap \mathbb{D}^{\mathbb{N}}\right)\right)$ and the (old) abscissa of uniform convergence $\sigma_{u}$.

Definition 2.4. Given $1 \leq w \leq \infty$ we will say that a set of holomorphic functions $\mathcal{F}\left(\ell_{w} \cap \mathbb{D}^{\mathbb{N}}\right)$ has the property $(T)$ if it remains stable under composition with diagonal operators of norm $\leq 1$, contains the continuous polynomials on $\ell_{w}$ as well as all bounded holomorphic functions on $\ell_{w} \cap \mathbb{D}^{\mathbb{N}}$.

Relevant examples are the space $H\left(\ell_{w} \cap \mathbb{D}^{\mathbb{N}}\right)$ of all holomorphic functions, the space $H_{b}\left(\ell_{w} \cap \mathbb{D}^{\mathbb{N}}\right)$ of holomorphic functions of bounded type, and the space $H_{\beta}\left(\ell_{w} \cap \mathbb{D}^{\mathbb{N}}\right)$ of all holomorphic functions that are bounded on bounded subsets of $\ell_{w} \cap \mathbb{D}^{\mathbb{N}}$ (see [14, p. 164] for the definition and properties). We have $H_{\infty}\left(B_{\ell_{\infty}}\right)=H_{\beta}\left(B_{\ell_{\infty}}\right)$, but $H_{\infty}\left(B_{\ell_{w}}\right) \subsetneq H_{\beta}\left(B_{\ell_{w}}\right) \subsetneq$ $H_{b}\left(B_{\ell_{w}}\right) \subsetneq H\left(B_{\ell_{w}}\right)$, for all $1 \leq w<+\infty$.

Theorem 2.5. Let $\sum_{n=1}^{\infty} \frac{a_{n}}{n^{s}}$ be a Dirichlet series. For any set $\mathcal{F}\left(\ell_{w} \cap\right.$ $\mathbb{D}^{\mathbb{N}}$ ) of holomorphic functions on $\ell_{w} \cap \mathbb{D}^{\mathbb{N}}$ with property $(T)$ we have

$$
h\left(\mathcal{F}\left(\ell_{w} \cap \mathbb{D}^{\mathbb{N}}\right)\right) \leq \sigma_{u} \leq h\left(\mathcal{F}\left(\ell_{w} \cap \mathbb{D}^{\mathbb{N}}\right)\right)+\frac{1}{w},
$$

for all $1 \leq w \leq \infty$.

Proof: This proof heavily relies on the original one by Bohr [6] (see also the version given by Boas in $[\mathbf{3}]$; the interested reader should also 
compare with Lemmas 17 and 18 from Bayart's paper [2]). But, as there are some delicate technical difficulties to overcome, we feel that we must give it with detail. By hypothesis, $H_{\infty}\left(\ell_{w} \cap \mathbb{D}^{\mathbb{N}}\right) \subset \mathcal{F}\left(\ell_{w} \cap\right.$ $\left.\mathbb{D}^{\mathbb{N}}\right)$, hence $h\left(\mathcal{F}\left(\ell_{w} \cap \mathbb{D}^{\mathbb{N}}\right)\right) \leq h\left(H_{\infty}\left(\ell_{w} \cap \mathbb{D}^{\mathbb{N}}\right)\right)$ and, by Proposition 2.3, $h\left(H_{\infty}\left(B_{\ell_{\infty}}\right)\right)=h\left(H_{\infty}\left(\ell_{w} \cap \mathbb{D}^{\mathbb{N}}\right)\right)$. But Bohr proved that $h\left(H_{\infty}\left(B_{\ell_{\infty}}\right)\right)=$ $\sigma_{u}$ (for a direct proof see e.g. [12, Theorem 4.4]) and we get $h\left(\mathcal{F}\left(\ell_{w} \cap\right.\right.$ $\left.\left.\mathbb{D}^{\mathbb{N}}\right)\right) \leq \sigma_{u}$.

To prove the other inequality consider $\sigma \in \mathbb{R}$ such that $g_{\sigma} \in \mathcal{F}\left(\ell_{w} \cap\right.$ $\left.\mathbb{D}^{\mathbb{N}}\right)$ with monomial expansion $\sum_{\alpha \in \mathbb{N}_{0}^{(\mathbb{N})}} \frac{a_{p^{\alpha}}}{p^{\alpha \sigma}} z^{\alpha}$. We can assume $\sigma=0$. This means that

$$
g(z):=\sum_{\alpha \in \mathbb{N}_{0}^{(\mathbb{N})}} a_{p^{\alpha}} z^{\alpha} \in \mathcal{F}\left(\ell_{w} \cap \mathbb{D}^{\mathbb{N}}\right) .
$$

To finish the proof we have to check that $\sigma_{u} \leq \frac{1}{w}$. For $m \in \mathbb{N}$, the $m$-dimensional unit polydisk $B_{\infty}^{m}=B_{\left(\mathbb{C}^{m},\|\cdot\|_{\infty}\right)}$ is a Reinhardt domain in $\mathbb{C}^{m}$ such that $B_{\infty}^{m} \times\{0\}=\ell_{w} \cap \mathbb{D}^{\mathbb{N}} \cap\left(\ell_{w}^{m} \times\{0\}\right)$. We define $g_{m}: B_{\infty}^{m} \longrightarrow$ $\mathbb{C}$ by $g_{m}\left(z_{1}, \ldots, z_{m}\right)=g\left(z_{1}, \ldots, z_{m}, 0, \ldots\right)$. Clearly, $g_{m} \in H\left(B_{\infty}^{m}\right)$. We put $A_{m}:=\left\{p^{\alpha}: \alpha \in \mathbb{N}_{0}^{m}\right\}$ and

$$
a_{n}^{(m)}:= \begin{cases}a_{n} & \text { if } n \in A_{m} \\ 0 & \text { otherwise }\end{cases}
$$

and since $0<\left|\frac{1}{p_{k}^{s}}\right|<1$ for all $k \in \mathbb{N}$ and all $s \in \mathbb{C}$ with $\operatorname{Re} s>0$, it is possible to define a sequence $\left(f_{m}\right)$ of holomorphic functions, $f_{m}$ : $[\operatorname{Re} s>$ $0] \longrightarrow \mathbb{C}, f_{m}(s)=g_{m}\left(\frac{1}{p_{1}^{s}}, \ldots, \frac{1}{p_{m}^{s}}\right)$. This sequence satisfies the following properties:

(1) $\left(f_{m}\right) \subset H([\operatorname{Re} s>0])$ and for each $m \in \mathbb{N}, f_{m}(s)=\sum_{n=1}^{\infty} \frac{a_{n}^{(m)}}{n^{s}}$, where the convergence is absolute for every $s \in \mathbb{C}$ with $\operatorname{Re} s>0$.

(2) Given $m$, the Dirichlet series $\sum_{n=1}^{\infty} \frac{a_{n}^{(m)}}{n^{s}}$ converges absolutely and uniformly on $[\operatorname{Re} s \geq \eta]$, for every $\eta>0$.

(3) $\left(f_{m}\right)$ is bounded on $[\operatorname{Re} s \geq \eta]$, for each $\eta>\frac{1}{w}$.

(4) For each $\eta>\frac{1}{w}$ the sequence $f_{m}(s)=\sum_{n \in A_{m}} \frac{a_{n}^{(m)}}{n^{s}}$ is absolutely and uniformly convergent to the Dirichlet series $\sum_{n=1}^{\infty} \frac{a_{n}}{n^{s}}$ on $[\operatorname{Re} s \geq$ $\eta+1]$.

Since $\sum_{\alpha \in \mathbb{N}_{0}^{m}} a_{p^{\alpha}} z^{\alpha}$ converges absolutely and uniformly to $g_{m}$ on every compact subset of $B_{\infty}^{m}$, (1) holds. 
Claim (2) follows from the fact that $K_{\eta}^{m}=\left\{z \in \mathbb{C}^{m}:\left|z_{n}\right| \leq p_{n}^{-\eta}, n=\right.$ $1,2, \ldots, m\}$ is a compact subset of $B_{\infty}^{m}$, and $K_{\eta}^{m}$ is the image by the mapping $s \rightarrow\left(\frac{1}{p_{1}^{s}}, \ldots, \frac{1}{p_{m}^{s}}\right)$ of $[\operatorname{Re} s \geq \eta]$.

Given $\eta>\frac{1}{w}$, the sequence $\left(\frac{1}{p^{\eta}}\right):=\left(\frac{1}{p_{k}^{\eta}}\right)_{k=1}^{\infty} \in \ell_{w} \cap \mathbb{D}^{\mathbb{N}}$, so $g\left(\left(\frac{1}{p^{\eta}}\right)\right)$ is defined. Let $K_{\eta}:=\left\{x \in \mathbb{C}^{\mathbb{N}}:\left|x_{k}\right| \leq p_{k}^{-\eta}, k=1,2, \ldots\right\}$. In $K_{\eta}$ the $\|\cdot\|_{w}$-norm topology coincides with the pointwise topology. Hence $K_{\eta}$ is a compact subset of $\ell_{w} \cap \mathbb{D}^{\mathbb{N}}$ containing $\left(\frac{1}{p_{k}^{\pi}}\right)$ (for the case $w=\infty$ observe that $\left.K_{\eta} \subset c_{0}\right)$. Finally, the inequalities

$$
\sup _{m \in \mathbb{N},[\operatorname{Re} s \geq \eta]}\left|f_{m}(s)\right| \leq \sup _{m \in \mathbb{N}, z \in K_{\eta}^{m}}\left|g_{m}(z)\right| \leq \sup _{x \in K_{\eta}}|g(x)|<\infty,
$$

imply that the sequence $\left(f_{m}\right)$ is bounded on $[\operatorname{Re} s \geq \eta]$ for every $\eta>\frac{1}{w}$ proving (3).

Finally fix $\eta>\frac{1}{w}$. For $\alpha \in \mathbb{N}_{0}^{(\mathbb{N})}$ and $m \in \mathbb{N}$ such that $\alpha_{n}=0$ for all $n>m$

$$
a_{p^{\alpha}}=\left(\frac{1}{2 \pi i}\right)^{m} \int_{\left|z_{1}\right|=p_{1}^{-\eta}} \ldots \int_{\left|z_{m}\right|=p_{m}^{-\eta}} \frac{g\left(z_{1}, \ldots, z_{m}, 0, \ldots\right)}{z_{1}^{\alpha_{1}+1} \ldots z_{m}^{\alpha_{m}+1}} d z_{1} \ldots d z_{m} .
$$

Thus

$$
\begin{aligned}
\left|a_{p^{\alpha}} \frac{1}{p^{\eta \alpha}}\right| \leq\left(\frac{1}{2 \pi}\right)^{m} \int_{[0,2 \pi]^{m}}\left|g\left(p_{1}^{-\eta} e^{i \theta_{1}}, \ldots, p_{m}^{-\eta} e^{i \theta_{m}}, 0, \ldots\right)\right| d \theta & \\
& \leq\|g\|_{K_{\eta}}<\infty
\end{aligned}
$$

and it follows that

$$
\left\{a_{n} \frac{1}{n^{\eta}}: n \in \mathbb{N}\right\}=\left\{a_{p^{\alpha}} \frac{1}{p^{\eta \alpha}}: \alpha \in \mathbb{N}_{0}^{(\mathbb{N})}\right\}
$$

is bounded. Since the sequence $\left(\frac{1}{n^{t}}\right)_{n=1}^{\infty} \in \ell_{1}$ for every $t>1$ we conclude that

$$
\left\{a_{n} \frac{1}{n^{\eta+1}}: n \in \mathbb{N}\right\} \in \ell_{1}
$$

for every $\eta>\frac{1}{w}$ and

$$
\left|a_{n} \frac{1}{n^{s}}\right| \leq\left|a_{n}\right| \frac{1}{n^{\eta+1}},
$$

for all $s$ such that Re $s \geq \eta+1$, which proves the claim (4).

By (1), (2) and (3) and Montel Theorem, there exists a holomorphic function $f$ on $\left[\operatorname{Re} s>\frac{1}{w}\right]$ such that $\left(f_{m}\right)$ converges absolutely and 
uniformly to $f$ on any compact subset of $\left[\operatorname{Re} s>\frac{1}{w}\right]$ and, by (4),

$$
f(s)=\sum_{n=1}^{\infty} \frac{a_{n}}{n^{s}},
$$

for all $s$ in $[\operatorname{Re} s \geq \eta+1]$. Moreover, by (3), $f$ is bounded on $[\operatorname{Re} s \geq \eta]$.

Our next goal, that will finish the proof, is to show that $f(s)=$ $\sum_{n=1}^{\infty} \frac{a_{n}}{n^{s}}$ for all $s \in \mathbb{C}$ with $\operatorname{Re} s>\frac{1}{w}$ and that the convergence is uniform on $[\operatorname{Re} s \geq \eta]$. But if we take a number $b$ so that $\frac{1}{w}<b<\eta<b+1$ and consider $\Omega=[\eta \leq \operatorname{Re} s \leq b+1]$ then $[\operatorname{Re} s \geq \eta]=\Omega \cup[\operatorname{Re} s \geq \eta+1]$. Thus, by (4), we only have to prove the uniform convergence of the Dirichlet series $\sum_{n=1}^{\infty} \frac{a_{n}}{n^{s}}$ on $\Omega$.

Let $s_{0}:=x_{0}+i y_{0} \in \Omega$. For $r>0$ take the closed path $\gamma_{r}$ given by the union of the following segments $\left[e_{1}, e_{2}\right],\left[e_{2}, e_{3}\right],\left[e_{3}, e_{4}\right],\left[e_{4}, e_{1}\right]$, where $e_{1}, e_{2}, e_{3}, e_{4}$ are the following points

$e_{1}=\eta+1+i\left(y_{0}-r\right), e_{2}=\eta+1+i\left(y_{0}+r\right), e_{3}=b+i\left(y_{0}+r\right), e_{4}=b+i\left(y_{0}-r\right)$.

Now we take a sequence $\left(d_{m}\right)$ of positive real numbers satisfying the two conditions:

(i) $d_{m}>n$ for $n=1, \ldots, m$ and $d_{m}<n$ for $n>m$,

(ii) there exists $K>0$ so that $\left(\min _{n \in \mathbb{N}}\left\{\left|\log \frac{d_{m}}{n}\right|\right\}\right)^{-1} \leq K m$, for all $m$.

Bohr took $d_{m}=\sqrt{m(m+1)}$. In that case, $\min _{n \in \mathbb{N}}\left\{\left|\log \frac{d_{m}}{n}\right|\right\}=$ $\log \sqrt{1+\frac{1}{m}}$.

Let us define $h_{m}:\left[\operatorname{Re} s>\frac{1}{w}\right] \longrightarrow \mathbb{C}$ by

$$
h_{m}(s)=\frac{d_{m}^{s-s_{0}}}{s-s_{0}} f(s) .
$$

So $f\left(s_{0}\right)=\operatorname{res}_{s-s_{0}} h_{m}=\frac{1}{2 \pi i} \int_{\gamma_{r}} h_{m}(s) d s$. Moreover,

$$
\begin{aligned}
2 \pi\left|f\left(s_{0}\right)-\sum_{n=1}^{m} \frac{a_{n}}{n^{s_{0}}}\right| & =\left|\int_{\gamma_{r}} h_{m}(s) d s-\sum_{n=1}^{m} \frac{a_{n}}{n^{s_{0}}}\right| \\
& \leq\left|\int_{e_{1}}^{e_{2}} h_{m}(s) d s-2 \pi i \sum_{n=1}^{m} \frac{a_{n}}{n^{s_{0}}}\right|+\left|\int_{e_{2}}^{e_{3}} h_{m}(s) d s\right| \\
& +\left|\int_{e_{3}}^{e_{4}} h_{m}(s) d s\right|+\left|\int_{e_{4}}^{e_{1}} h_{m}(s) d s\right|
\end{aligned}
$$


Let us see first what happens with $\int_{e_{1}}^{e_{2}} h_{m}(s) d s$. Since

$$
\left|\frac{d_{m}^{s-s_{0}}}{s-s_{0}}\right|=\frac{d_{m}^{\operatorname{Re}\left(s-s_{0}\right)}}{\left|s-s_{0}\right|}=\frac{d_{m}^{\eta+1-x_{0}}}{\left|s-s_{0}\right|} \leq \frac{d_{m}^{\eta+1}}{\eta-b},
$$

for all $s \in\left[e_{1}, e_{2}\right]$ and since the series $\sum_{n=1}^{\infty} \frac{a_{n}}{n^{s}}$ is uniformly convergent on $[\operatorname{Re} s=\eta+1]$, it follows that

$$
\int_{e_{1}}^{e_{2}} h_{m}(s) d s=\sum_{n=1}^{\infty} a_{n} \int_{e_{1}}^{e_{2}} \frac{d_{m}^{s-s_{0}}}{\left(s-s_{0}\right) n^{s}} d s=\sum_{n=1}^{\infty} \frac{a_{n}}{n^{s_{0}}} \int_{e_{1}-s_{0}}^{e_{2}-s_{0}}\left(\frac{d_{m}}{n}\right)^{s} \frac{1}{s} d s .
$$

Therefore

$$
\begin{aligned}
\left|\int_{e_{1}}^{e_{2}} h_{m}(s) d s-2 \pi i \sum_{n=1}^{m} \frac{a_{n}}{n^{s_{0}}}\right| \leq & \left|\sum_{n=1}^{m} \frac{a_{n}}{n^{s_{0}}}\left(\int_{e_{1}-s_{0}}^{e_{2}-s_{0}}\left(\frac{d_{m}}{n}\right)^{s} \frac{1}{s} d s-2 \pi i\right)\right| \\
& +\sum_{n>m}\left|\frac{a_{n}}{n^{s_{0}}}\right|\left|\int_{e_{1}-s_{0}}^{e_{2}-s_{0}}\left(\frac{d_{m}}{n}\right)^{s} \frac{1}{s} d s\right| .
\end{aligned}
$$

Now, if $n \leq m$ then $\frac{d_{m}}{n}>1$ and hence by [15, pp. 825-826]

$$
\left|\int_{e_{1}-s_{0}}^{e_{2}-s_{0}}\left(\frac{d_{m}}{n}\right)^{s} \frac{1}{s} d s-2 \pi i\right| \leq \frac{2}{r} \frac{\left(\frac{d_{m}}{n}\right)^{a}}{\log \left(\frac{d_{m}}{n}\right)}
$$

and if $n>m$ then $0<\frac{d_{m}}{n}<1$ and if we apply again [15, pp. 825-826] getting

$$
\left|\int_{e_{1}-s_{0}}^{e_{2}-s_{0}}\left(\frac{d_{m}}{n}\right)^{s} \frac{1}{s} d s\right| \leq \frac{2}{r} \frac{\left(\frac{d_{m}}{n}\right)^{a}}{-\log \left(\frac{d_{m}}{n}\right)},
$$

where $0<a:=\eta+1-x_{0} \leq 1$. Hence

$$
\left|\int_{e_{1}}^{e_{2}} h_{m}(s) d s-2 \pi i \sum_{n=1}^{m} \frac{a_{n}}{n^{s_{0}}}\right| \leq \sum_{n=1}^{\infty} \frac{\left|a_{n}\right|}{n^{x_{0}}} \frac{2}{r} \frac{\left(\frac{d_{m}}{n}\right)^{a}}{\left|\log \left(\frac{d_{m}}{n}\right)\right|} .
$$

By hypothesis, there exists $K>0$ so that

$$
\frac{1}{\left|\log d_{m}-\log n\right|} \leq K m
$$

for all $m$ and $n$. Thus,

$$
\left|\int_{e_{1}}^{e_{2}} h_{m}(s) d s-2 \pi i \sum_{n=1}^{m} \frac{a_{n}}{n^{s_{0}}}\right| \leq \frac{2 K m}{r} d_{m}^{a} \sum_{n=1}^{\infty} \frac{\left|a_{n}\right|}{n^{\eta+1}} .
$$


Hence, since $a \leq 1$ and, by (2.3), we finally have that there is $C_{1}>0$ such that

$$
\left|\int_{e_{1}}^{e_{2}} h_{m}(s) d s-2 \pi i \sum_{n=1}^{m} \frac{a_{n}}{n^{s_{0}}}\right| \leq C_{1} \frac{m d_{m}}{r}
$$

for all $m$ and $r>0$. Now

$\left|\int_{e_{2}}^{e_{3}} h_{m}(s) d s\right|=\left|\int_{e_{2}-s_{0}}^{e_{3}-s_{0}} \frac{d_{m}^{s}}{s} f\left(s+s_{0}\right) d s\right| \leq \sup _{[\operatorname{Re} s \geq b]}|f(s)| \int_{b-x_{0}}^{\eta+1-x_{0}} \frac{d_{m}^{t}}{|t+i r|} d t$ and, as $0 \leq \eta+1-x_{0} \leq 1$, we get a constant $C_{2}=(\eta+1-b) \sup _{[\operatorname{Re} s \geq b]}|f(s)|$ such that

$$
\left|\int_{e_{2}}^{e_{3}} h_{m}(s) d s\right| \leq C_{2} \frac{d_{m}}{r}
$$

for all $m$ and $r>0$. Analogously, there exists a constant $C_{3}$ so that

$$
\left|\int_{e_{4}}^{e_{1}} h_{m}(s) d s\right| \leq C_{3} \frac{d_{m}}{r}
$$

for all $m$ and $r>0$. Finally

$$
\begin{aligned}
\left|\int_{e_{3}}^{e_{4}} h_{m}(s) d s\right| & =\left|\int_{e_{3}-s_{0}}^{e_{4}-s_{0}} \frac{d_{m}^{s}}{s} f\left(s+s_{0}\right) d s\right| \\
& \leq \sup _{[\operatorname{Re} s \geq b]}|f(s)| d_{m}^{b-x_{0}} \int_{-r}^{r} \frac{2}{\left|b-x_{0}+t i\right|} d t \\
& \leq 4 \sup _{[\operatorname{Re} s \geq b]}|f(s)| d_{m}^{b-x_{0}} \int_{0}^{r} \frac{1}{\eta-b+t} d t \\
& =4 \sup _{[\operatorname{Re} s \geq b]}|f(s)| d_{m}^{b-x_{0}}(\log (\eta-b+r)-\log (\eta-b)) .
\end{aligned}
$$

Hence, there exists $C_{4}>0$ so that

$$
\left|\int_{e_{3}}^{e_{4}} h_{m}(s) d s\right| \leq C_{4} \frac{\log r}{d_{m}^{\eta-b}}
$$

for all $m \in \mathbb{N}$ and all $r>1$. If we take $r:=m d_{m}^{2}<d_{m}^{3}$, by applying (2.4), (2.5), (2.6) and (2.7), we obtain that there is a constant $C>0$ with

$$
\left|f\left(s_{0}\right)-\sum_{n=1}^{m} \frac{a_{n}}{n^{s_{0}}}\right| \leq C\left(\frac{1}{d_{m}}+\frac{3 \log d_{m}}{d_{m}^{\eta-b}}\right)
$$

for all $s_{0} \in \Omega$ and all $m \in \mathbb{N}$ and it follows that the Dirichlet series converges uniformly to $f$ in $\Omega$. 
We finish this section with three examples in which $\sigma_{u}$ in fact equals $h\left(\mathcal{F}\left(\ell_{w} \cap \mathbb{D}^{\mathbb{N}}\right)\right)+\frac{1}{w}$. A natural conjecture might be that $\sigma_{u}=h\left(\mathcal{F}\left(\ell_{w} \cap\right.\right.$ $\left.\left.\mathbb{D}^{\mathbb{N}}\right)\right)+\frac{1}{w}$. But this is in general false as will be shown at the end of the next section.

Examples 2.6. In the following we abbreviate for a given $1 \leq w \leq \infty$ the abscissa $h\left(\mathcal{F}\left(\ell_{w} \cap \mathbb{D}^{\mathbb{N}}\right)\right)$ in the three cases $h\left(H\left(\ell_{w} \cap \mathbb{D}^{\mathbb{N}}\right)\right), h\left(H_{\beta}\left(\ell_{w} \cap\right.\right.$ $\left.\left.\mathbb{D}^{\mathbb{N}}\right)\right)$ and $h\left(H_{b}\left(\ell_{w} \cap \mathbb{D}^{\mathbb{N}}\right)\right)$ simply by $h_{w}$.

(i) For the Riemann zeta function $\sum_{n=1}^{\infty} \frac{1}{n^{s}}$ holds that $\sigma_{u}=\sigma_{a}=1$ and $h_{w}=1-\frac{1}{w}$ for every $1 \leq w \leq \infty$. In particular, $h_{\infty}=\sigma_{u}=$ $\sigma_{a}=1$.

(ii) For the Dirichlet series $\sum_{n=1}^{\infty} \frac{a_{n}}{n^{s}}$, where $a_{n}=1$ if $n$ is a prime number and 0 otherwise, holds that $\sigma_{u}=\sigma_{a}=1$ and $h_{w}=1-\frac{1}{w}$ for every $1 \leq w \leq \infty$. In particular, $h_{\infty}=\sigma_{u}=1$.

(iii) For the Dirichlet series $\sum_{n=1}^{\infty} \frac{1}{\left(p_{1} \ldots p_{n}\right)^{s}}$ holds that $\sigma_{a}=\sigma_{u}=0$ and $h_{w}=-\frac{1}{w}$ for every $1 \leq w \leq \infty$.

Proof: We start with the proof of (i): For $1<w<\infty$ we just notice that for $\sigma \in \mathbb{R}$ we have

$$
\left|\sum_{\alpha \in \mathbb{N}_{0}^{(N)}} \frac{1}{p^{\sigma \alpha}} z^{\alpha}\right| \leq\left(\sum_{\alpha \in \mathbb{N}_{0}^{(N)}} \frac{1}{p^{\sigma w^{\prime} \alpha}}\right)^{\frac{1}{w^{\prime}}}\left(\sum_{\alpha \in \mathbb{N}_{0}^{(N)}}\left|z^{w \alpha}\right|\right)^{\frac{1}{w}},
$$

where $\frac{1}{w^{\prime}}=1-\frac{1}{w}$. Moreover $\sum_{\alpha \in \mathbb{N}_{0}^{(\mathbb{N})}}\left|z^{w \alpha}\right|=\prod_{n=1}^{\infty} \frac{1}{1-\left|z_{n}\right| w}$ and this product converges for $z \in \ell_{w} \cap \mathbb{D}^{\mathbb{N}}$ and, on the other hand, $\sum_{\alpha \in \mathbb{N}_{0}^{(N)}} \frac{1}{p^{\sigma w^{\prime} \alpha}}=$ $\prod_{n=1}^{\infty} \frac{1}{1-\left|\frac{1}{p_{n}^{\sigma}}\right|^{w^{\prime}}}$ and this product converges if and only if $\sum_{n=1}^{\infty} \frac{1}{\left(p_{n}^{\sigma}\right)^{w^{\prime}}}<$ $+\infty$, but this holds if and only if $\sigma>\frac{1}{w^{\prime}}$.

The proof of (ii) is left to the reader.

Finally, the proof of (iii): Given $\sigma>0$, clearly $p_{1}^{\sigma}>1$. Thus,

$$
\left|\frac{1}{\left(p_{1} \ldots p_{n}\right)^{s}}\right| \leq\left(\frac{1}{p_{1}^{\sigma}}\right)^{n}
$$

for every natural number $n \geq k_{0}$ and every $s \in \mathbb{C}$ with $\operatorname{Re} s \geq \sigma$. Hence

$$
\sum_{n=1}^{\infty}\left|\frac{1}{\left(p_{1} \ldots p_{n}\right)^{s}}\right|<\infty
$$


Therefore $\sigma_{a} \leq 0$. But, for $s \in \mathbb{C}$ with $\operatorname{Re} s<0$ it follows that $\left|\left(p_{1} \ldots p_{n}\right)^{-s}\right|=\left(p_{1} \ldots p_{n}\right)^{-\operatorname{Re} s}$ which diverges to $+\infty$, so $\sigma_{c} \geq 0$. Then we get that $0 \leq \sigma_{c} \leq \sigma_{u} \leq \sigma_{a} \leq 0$ which implies that $\sigma_{c}=\sigma_{u}=\sigma_{a}=0$. But $\left|z_{n}\right|<1$ for all $n$ and all $z \in \ell_{w} \cap \mathbb{D}^{\mathbb{N}}$, hence (2.8) implies that $\sum_{n=1}^{\infty} \frac{z_{1} \ldots z_{n}}{p_{1}^{\sigma} \ldots p_{n}^{\sigma}} \in H_{\infty}\left(\ell_{w} \cap \mathbb{D}^{\mathbb{N}}\right)$ for every $\sigma>0$. Therefore $h_{w} \leq 0$. Now, let $\sigma<0$. For $1 \leq w<\infty$, by [14, Lemma 1.38],

$$
\frac{\left\|z_{1} \ldots z_{n}\right\|_{r B_{\ell_{w}}}}{p_{1}^{\sigma} \ldots p_{n}^{\sigma}}=r^{n} \frac{p_{1}^{-\sigma} \ldots p_{n}^{-\sigma}}{n^{\frac{n}{w}}}
$$

for all $r>0$. Putting $b_{n, r}:=r^{n} \frac{p_{1}^{-\sigma} \ldots p_{n}^{-\sigma}}{n^{\frac{n}{w}}}$, we have that

$$
\frac{b_{n+1, r}}{b_{n, r}}=r\left(\frac{n}{n+1}\right)^{\frac{n}{w}} \frac{p_{n+1}^{-\sigma}}{(n+1)^{\frac{1}{w}}} .
$$

On one hand, the sequence $\left(\left(\frac{n}{n+1}\right)^{\frac{n}{w}}\right)$ converges to $e^{-\frac{1}{w}}$ and, on the other hand, since $K n \log (n)<p_{n}<L n \log (n)$ for every $n \geq 2$ and some positive constants $K$ and $L$, the sequence $\left(\frac{p_{n+1}^{-w \sigma}}{n+1}\right)^{\frac{1}{w}}$ diverges to $+\infty$ whenever $\sigma \leq-\frac{1}{w}$ and converges to 0 whenever $\sigma>-\frac{1}{w}$. Thus, if $\sigma>-\frac{1}{w}$, then the series $\sum_{n=1}^{\infty} b_{n, r}$ is convergent and, hence, $f_{\sigma}=$ $\sum_{n=1}^{\infty} \frac{z_{1} \ldots z_{n}}{p_{1}^{\sigma} \ldots p_{n}^{\sigma}}$ is holomorphic and bounded on $r B_{\ell_{w}} \cap \mathbb{D}$ for all $r>0$, which implies that $f_{\sigma}$ belongs to the smallest space $H_{\beta}\left(\ell_{w} \cap \mathbb{D}^{\mathbb{N}}\right)$ and we have $h_{w} \leq-\frac{1}{w}$ for the three spaces.

Now, given $\sigma \leq-\frac{1}{w}$ we have that $b_{n, r}$ diverges to $\infty$ for all $r>0$. If $f_{\sigma}=\sum_{n=1}^{\infty} \frac{z_{1} \ldots z_{n}}{p_{1}^{\sigma} \ldots p_{n}^{\sigma}}$ belongs to the biggest space $H\left(\ell_{w} \cap \mathbb{D}^{\mathbb{N}}\right)$ then, by continuity at the origin, there would exists $r_{0}>0$ such that $f_{\sigma} \in$ $H_{\infty}\left(r_{0} B_{\ell_{w}}\right)$. But, by the Cauchy inequalities,

$$
b_{n, r_{0}}=\frac{\left\|z_{1} \ldots z_{n}\right\|_{r_{0} B_{\ell_{w}}}}{p_{1}^{\sigma} \ldots p_{n}^{\sigma}} \leq\left\|f_{\sigma}\right\|_{r_{0} B_{\ell_{w}}}<\infty
$$

for all $n$, a contradiction. Therefore $h_{w}=-\frac{1}{w}$. The case $w=\infty$ also follows since $\left(p_{1}^{-\sigma} \ldots p_{n}^{-\sigma}\right)$ diverges to $+\infty$.

\section{3. $S_{w}$ versus $T_{w}$}

Let $R$ be a Reinhardt domain in $\ell_{w}$, for $1 \leq w \leq \infty$, and $\mathcal{F}(R)$ a set of holomorphic functions on $R$. We define

$$
S(\mathcal{F}(R)):=\sup \left\{q \geq 1: \ell_{q} \cap R \subset \operatorname{dom} \mathcal{F}(R)\right\}
$$

and

$$
T(\mathcal{F}(R)):=\sup \left\{\sigma_{a}-h(\mathcal{F}(R))\right\}
$$


where this supremum runs over all Dirichlet series $\sum_{n=1}^{\infty} \frac{a_{n}}{n^{s}}$ (as usual we use the notation $a-(+\infty)=-\infty, a-(-\infty)=+\infty,(+\infty)-b=+\infty$, $(-\infty)-b=-\infty$, for all $a, b \in \mathbb{R},+\infty-(+\infty)=0$ and $-\infty-(-\infty)=0)$.

Let us first note that for $\mathcal{F}(R)=H_{\infty}\left(\ell_{w} \cap \mathbb{D}^{\mathbb{N}}\right)$ we obtain nothing else than Bohr's original definitions from (1.2) and (1.4).

Proposition 3.1. $S\left(H_{\infty}\left(\ell_{w} \cap \mathbb{D}^{\mathbb{N}}\right)\right)=S$ and $T\left(H_{\infty}\left(\ell_{w} \cap \mathbb{D}^{\mathbb{N}}\right)\right)=T$ for all $1 \leq w \leq \infty$.

Proof: By Proposition 2.3, $T\left(H_{\infty}\left(\ell_{w} \cap \mathbb{D}^{\mathbb{N}}\right)\right)=T$ and an easy analysis of the proof of Proposition 2.3 shows that $S\left(H_{\infty}\left(\ell_{w} \cap \mathbb{D}^{\mathbb{N}}\right)\right)=S$ for all $1 \leq w \leq \infty$.

In particular, we know from (1.3) that $T\left(H_{\infty}\left(\ell_{w} \cap \mathbb{D}^{\mathbb{N}}\right)\right)=1 / S\left(H_{\infty}\left(\ell_{w} \cap\right.\right.$ $\left.\mathbb{D}^{\mathbb{N}}\right)$ ) for all $1 \leq w \leq \infty$ and, as a consequence, the Bohr-BohnenblustHille Theorem $T=1 / S=1 / 2$, i.e. the width of the strip of uniform but non absolute convergence for Dirichlet series is $\frac{1}{2}$, can actually be obtained from the study made on $\operatorname{dom}\left(H_{\infty}\left(\ell_{w} \cap \mathbb{D}^{\mathbb{N}}\right)\right)$ for any fixed $w$. But as we are going to show the situation is very different if we work with bigger classes of functions.

The following result is the main contribution in this section. It shows in which sense the Bohr-Bohnenblust-Hille Theorem can be extended from $H_{\infty}\left(B_{\ell_{\infty}}\right)$ to any set $\mathcal{F}\left(\ell_{w} \cap \mathbb{D}^{\mathbb{N}}\right)$ of holomorphic functions on $\ell_{w} \cap \mathbb{D}^{\mathbb{N}}$ satisfying property $(\mathrm{T})$.

Let us observe that for $1 \leq w<\infty$ the set $\ell_{w} \cap \mathbb{D}^{\mathbb{N}}$ is not bounded in $\ell_{w}$. Hence the function space $H_{\infty}\left(\ell_{w} \cap \mathbb{D}^{\mathbb{N}}\right)$ does not contain the dual space of $\ell_{w}$, and therefore next theorem does not apply to $H_{\infty}\left(\ell_{w} \cap \mathbb{D}^{\mathbb{N}}\right)$ (which fits with the preceding proposition).

Theorem 3.2. For $1 \leq w \leq \infty$ let $\mathcal{F}\left(\ell_{w} \cap \mathbb{D}^{\mathbb{N}}\right)$ be a set of holomorphic functions that contains the polynomials on $\ell_{w}$. Then we have

$$
S\left(\mathcal{F}\left(\ell_{w} \cap \mathbb{D}^{\mathbb{N}}\right)\right)= \begin{cases}1 & \text { if } 1 \leq w<2 \\ \left(\frac{1}{2}+\frac{1}{w}\right)^{-1} & \text { if } 2 \leq w \leq \infty,\end{cases}
$$

and if $\mathcal{F}\left(\ell_{w} \cap \mathbb{D}^{\mathbb{N}}\right)$ even satisfies property $(T)$

$$
T\left(\mathcal{F}\left(\ell_{w} \cap \mathbb{D}^{\mathbb{N}}\right)\right)=\frac{1}{S\left(\mathcal{F}\left(\ell_{w} \cap \mathbb{D}^{\mathbb{N}}\right)\right)}= \begin{cases}1 & \text { if } 1 \leq w<2 \\ \frac{1}{2}+\frac{1}{w} & \text { if } 2 \leq w \leq \infty .\end{cases}
$$

Proof: Clearly, (3.1) is an immediate consequence of Theorem 1.1. For the proof of (3.2) note first that by the Prime Number Theorem there exists a constant $K>0$ such that $n \leq p_{n} \leq K n \log n$ for all $n \geq 2$. Hence the series $\sum_{n=1}^{\infty} \frac{1}{p_{n}^{t}}$ is convergent if and only if $t>1$. Moreover, in order 
to make the notation short we will write $T_{w}=T\left(\mathcal{F}\left(\ell_{w} \cap \mathbb{D}^{\mathbb{N}}\right)\right), S_{w}=$ $S\left(\mathcal{F}\left(\ell_{w} \cap \mathbb{D}^{\mathbb{N}}\right)\right)$ and $h_{w}=h\left(\mathcal{F}\left(\ell_{w} \cap \mathbb{D}^{\mathbb{N}}\right)\right)$ for any Dirichlet series $\sum_{n=1}^{\infty} \frac{a_{n}}{n^{s}}$. Finally, note that by Proposition 2.1 we only have to study the case when $-\infty<h_{w}<+\infty$.

We start with the proof of the fact that $T_{w} \leq \frac{1}{S_{w}}$. Fix some Dirichlet series $\sum_{n=1}^{\infty} \frac{a_{n}}{n^{s}}$. If $1 \leq w \leq 2$ we know from (3.1) that $S_{w}=1$. We take $\sigma>h_{w}+1$ and $\sigma_{0}$ such that $\sigma-1>\sigma_{0}>h_{w}$. We know that

$$
g_{\sigma_{0}}(z)=\sum_{\alpha \in \mathbb{N}_{0}^{(\mathbb{N})}} \frac{a_{p^{\alpha}}}{p^{\alpha \sigma_{0}}} z^{\alpha} \in \mathcal{F}\left(\ell_{w} \cap \mathbb{D}^{\mathbb{N}}\right) .
$$

Since $\sigma-\sigma_{0}>1$, the sequence $\left(\frac{1}{p^{\sigma-\sigma_{0}}}\right)=\left(\frac{1}{p_{n}^{\sigma-\sigma_{0}}}\right)_{n=1}^{\infty}$ belongs to $\ell_{1} \cap B_{\ell_{\infty}}$ which, by Theorem 1.1, is contained in $\operatorname{dom} \mathcal{F}\left(\ell_{w} \cap \mathbb{D}^{\mathbb{N}}\right)$. Hence

$$
\sum_{\alpha \in \mathbb{N}_{0}^{(\mathbb{N})}}\left|\frac{a_{p^{\alpha}}}{p^{\alpha \sigma}}\right|=\sum_{\alpha \in \mathbb{N}_{0}^{(\mathbb{N})}}\left|\frac{a_{p^{\alpha}}}{p^{\alpha \sigma_{0}}}\right|\left(\frac{1}{p^{\sigma-\sigma_{0}}}\right)^{\alpha}<+\infty,
$$

and we conclude that $\sigma_{a} \leq \sigma$ for all $\sigma>h_{w}+1$. Thus $\sigma_{a} \leq h_{w}+1$, or in other words $\sigma_{a}-h_{w} \leq \frac{1}{S_{w}}$. This for $1 \leq w \leq 2$ proves the inequality $T_{w} \leq \frac{1}{S_{w}}$.

If $w>2$, then, by (3.1), $S_{w}=\left(\frac{1}{2}+\frac{1}{w}\right)^{-1}>1$. Fix again some Dirichlet series $\sum_{n=1}^{\infty} \frac{a_{n}}{n^{s}}$. If $\sigma>h_{w}+\frac{1}{S_{w}}$, we take $\delta>0$ such that $\sigma \geq h_{w}+\frac{1}{S_{w}}+\delta$ and $\frac{1}{S_{w}}+\frac{\delta}{3}<1$. Additionally we define $q=\left(\frac{1}{S_{w}}+\frac{\delta}{3}\right)^{-1}$ and $r=\frac{1}{S_{w}}+\frac{2 \delta}{3}$. By definition, $q>1$ and $r q>1$. Hence $\left(p_{n}^{-r}\right) \in \ell_{q}$. But $q<S_{w}$ and $S_{w}=\left(\frac{1}{2}+\frac{1}{w}\right)^{-1}<w$. Thus $\left(p_{n}^{-r}\right) \in \ell_{w}$ and we have that $\left(p_{n}^{-r}\right)$ belongs to $\ell_{w} \cap \mathbb{D}^{\mathbb{N}} \cap \ell_{q}$ which in turn is contained in $\operatorname{dom} \mathcal{F}\left(\ell_{w} \cap \mathbb{D}^{\mathbb{N}}\right)$. On the other hand, since $\sigma_{0}:=h_{w}+\frac{\delta}{3}>h_{w}$ we get that $g_{\sigma_{0}}(z)=\sum_{\alpha \in \mathbb{N}_{0}^{(\mathbb{N})}} \frac{a_{p} \alpha}{p^{\alpha \sigma_{0}}} z^{\alpha} \in \mathcal{F}\left(\ell_{w} \cap \mathbb{D}^{\mathbb{N}}\right)$, hence

$$
\sum_{\alpha \in \mathbb{N}_{0}^{(\mathbb{N})}}\left|\frac{a_{p^{\alpha}}}{p^{\alpha\left(\sigma_{0}+r\right)}}\right|=\sum_{\alpha \in \mathbb{N}_{0}^{(\mathbb{N})}}\left|\frac{a_{p^{\alpha}}}{p^{\alpha \sigma_{0}}}\right|\left(\frac{1}{p^{r}}\right)^{\alpha}<\infty .
$$

Finally, $\sigma_{0}+r=h_{w}+\frac{1}{S_{w}}+\delta \leq \sigma$, and we see that as desired

$$
\sum_{\alpha \in \mathbb{N}_{0}^{(\mathbb{N})}}\left|\frac{a_{p^{\alpha}}}{p^{\alpha \sigma}}\right|<\infty
$$

This finishes the proof of the inequality $T_{w} \leq \frac{1}{S_{w}}$. 
Now we want to prove that $\frac{1}{S_{w}} \leq T_{w}$ for all $1 \leq w \leq \infty$. The first aim is to show that there is some $\delta_{0}>0$ such that for all $t>0$ with $S_{w}<1 / t<\delta_{0}$ we have

$$
\left(p_{n}^{-t}\right) \notin \operatorname{dom} \mathcal{F}\left(\ell_{w} \cap \mathbb{D}^{\mathbb{N}}\right) ;
$$

indeed, if $w=1$ we fix $0<t<1$ and we take any $r>1$ with $1=S_{1}<$ $r<\frac{1}{t}$. Since $t r<1$, then $\left(p_{n}^{-t}\right) \notin \ell_{r}$. If $w>1$ we consider $t>0$ such that $S_{w}<\frac{1}{t}<w$ and any $r$ with $S_{w}<r<\frac{1}{t}$. Since $w t>1$, we have $\left(p_{n}^{-t}\right) \in \ell_{w}$ and clearly $0<p_{n}^{-t}<1$ for all $n$. Thus $\left(p_{n}^{-t}\right) \in \ell_{w} \cap \mathbb{D}^{\mathbb{N}}$. But since $t r<1$, then $\left(p_{n}^{-t}\right) \notin \ell_{r}$, for any $r$ with $S_{w}<r<\frac{1}{t}$. If we apply in both cases the upper bounds given by Theorem 1.1, we obtain that $\left(p_{n}^{-t}\right) \notin \operatorname{dom} \mathcal{F}\left(\ell_{w} \cap \mathbb{D}^{\mathbb{N}}\right)$, the desired claim.

Hence there exists $g(z)=\sum_{\alpha \in \mathbb{N}_{0}^{(\mathbb{N})}} c_{\alpha} z^{\alpha} \in \mathcal{F}\left(\ell_{w} \cap \mathbb{D}^{\mathbb{N}}\right)$ such that

$$
\sum_{\alpha \in \mathbb{N}_{0}^{(\mathbb{N})}}\left|c_{\alpha}\right|\left(p_{n}^{-t}\right)^{\alpha}=+\infty .
$$

If we define now

$$
a_{n}= \begin{cases}c_{\alpha} & \text { if } n=p^{\alpha} \\ c_{0} & \text { if } n=1\end{cases}
$$

then

$$
\sum_{n=1}^{\infty}\left|\frac{a_{n}}{n^{t}}\right|=\sum_{\alpha \in \mathbb{N}_{0}^{(\mathbb{N})}}\left|c_{\alpha}\right|\left(p_{n}^{-t}\right)^{\alpha}=+\infty .
$$

As a consequence $\sigma_{a} \geq t$. But for that Dirichlet series we have $h_{w} \leq 0$. Indeed, $\sum_{\alpha \in \mathbb{N}_{0}^{(\mathbb{N})}} \frac{a_{p}^{\alpha}}{p^{\alpha 0}} z^{\alpha}$ coincides with $g$ on $\ell_{w} \cap \mathbb{D}^{\mathbb{N}}$ and, by hypothesis, $g \in \mathcal{F}\left(\ell_{w} \cap \mathbb{D}^{\mathbb{N}}\right)$. Now $\sigma_{a}-h_{w} \geq \sigma_{a} \geq t$ for all $t<\frac{1}{S_{w}}$ and we conclude that $\sigma_{a}-h_{w} \geq \frac{1}{S_{w}}$, for that particular Dirichlet series. Hence $T_{w}=$ $\sup \left\{\sigma_{a}-h_{w}\right\} \geq \frac{1}{S_{w}}$.

Observe that taking $w=\infty$ we have obtained a new proof of the Bohr-Bohnenblust-Hille Theorem.

Remark 3.3. A natural conjecture, reinforced by the Examples 2.6 is that $\sigma_{u}=h\left(\mathcal{F}\left(\ell_{w} \cap \mathbb{D}^{\mathbb{N}}\right)\right)+\frac{1}{w}$, for each set of functions $\mathcal{F}\left(\ell_{w} \cap \mathbb{D}^{\mathbb{N}}\right)$ with property $(\mathrm{T})$. But, in general, this conjecture is false since for each $1 \leq w<2$ and for each set of functions $\mathcal{F}\left(\ell_{w} \cap \mathbb{D}^{\mathbb{N}}\right)$ with property $(T)$ there exists a Dirichlet series $\sum_{n=1}^{\infty} \frac{a_{n}}{n^{s}}$ such that $\sigma_{u}<h\left(\mathcal{F}\left(\ell_{w} \cap \mathbb{D}^{\mathbb{N}}\right)\right)+\frac{1}{w}$. Indeed, if there is a set of functions $\mathcal{F}\left(\ell_{w} \cap \mathbb{D}^{\mathbb{N}}\right)$ with property (T) satisfying that 
conjecture for all Dirichlet series $\sum_{n=1}^{\infty} \frac{a_{n}}{n^{s}}$, then

$\sup \left\{\sigma_{a}-\left(h\left(\mathcal{F}\left(\ell_{w} \cap \mathbb{D}^{\mathbb{N}}\right)\right)+\frac{1}{w}\right): \sum_{n=1}^{\infty} \frac{a_{n}}{n^{s}}\right\}=\sup \left\{\sigma_{a}-\sigma_{u}: \sum_{n=1}^{\infty} \frac{a_{n}}{n^{s}}\right\}=\frac{1}{2}$,

by the Bohr-Bohnenblust-Hille Theorem ([5], [7]). Thus

$$
T\left(\mathcal{F}\left(\ell_{w} \cap \mathbb{D}^{\mathbb{N}}\right)\right)-\frac{1}{w}=\frac{1}{2},
$$

for every $1 \leq w \leq+\infty$ but, by Theorem 3.2 , we know that $T\left(\mathcal{F}\left(\ell_{w} \cap\right.\right.$ $\left.\left.\mathbb{D}^{\mathbb{N}}\right)\right)=1$ for every $1 \leq w<2$. A contradiction.

\section{References}

[1] R. M. Aron And P. D. Berner, A Hahn-Banach extension theorem for analytic mappings, Bull. Soc. Math. France 106(1) (1978), $3-24$.

[2] F. Bayart, Hardy spaces of Dirichlet series and their composition operators, Monatsh. Math. 136(3) (2002), 203-236.

[3] H. P. BoAs, Majorant series, Several complex variables (Seoul, 1998), J. Korean Math. Soc. 37(2) (2000), 321-337.

[4] H. P. Boas and D. Khavinson, Bohr's power series theorem in several variables, Proc. Amer. Math. Soc. 125(10) (1997), 2975-2979.

[5] H. F. Bohnenblust and E. Hille, On the absolute convergence of Dirichlet series, Ann. of Math. (2) 32(3) (1931), 600-622.

[6] H. BoHR, Uber die gleichmassige Konvergenz Dirichcetscher Reihen, J. Reine Angew. Math. 143 (1913), 203-211.

[7] H. Bohr, Uber die Bedeutung der Potenzreihen unendlich vieler Variabeln in der Theorie der Dirichletschen Reihen $\sum \frac{a_{n}}{n^{s}}$, Nachr. Akad. Wiss. Göttingen Math.-Phys. Kl. (1913), 441-448.

[8] H. Bohr, A theorem concerning power series, Proc. London Math. Soc. (2) 13 (1914), 1-5.

[9] A. M. Davie and T. W. Gamelin, A theorem on polynomial-star approximation, Proc. Amer. Math. Soc. 106(2) (1989), 351-356.

[10] A. Defant, D. García, and M. Maestre, Bohr's power series theorem and local Banach space theory, J. Reine Angew. Math. 557 (2003), 173-197.

[11] A. Defant, D. García, and M. Maestre, Estimates for the first and second Bohr radii of Reinhardt domains, J. Approx. Theory 128(1) (2004), 53-68. 
[12] A. Defant, D. García, M. Maestre, and D. Pérez-García, Bohr's strip for vector valued Dirichlet series, Math. Ann. 342(3) (2008), 533-555.

[13] A. Defant, M. Maestre, and C. Prengel, Domains of convergence for monomial expansions of holomorphic functions in infinitely many variables, J. Reine Angew. Math. 634 (2009), 13-49.

[14] S. Dineen, "Complex analysis on infinite-dimensional spaces", Springer Monographs in Mathematics, Springer-Verlag London, Ltd., London, 1999.

[15] E. Landau, "Handbuch der Lehre von der Verteilung der Primzahlen", Vol. II, Leipzig und Berlin, B. G. Teubner, 1909.

[16] J. MujicA, "Complex analysis in Banach spaces", Holomorphic functions and domains of holomorphy in finite and infinite dimensions, North-Holland Mathematics Studies 120, Notas de Matemática [Mathematical Notes] 107, North-Holland Publishing Co., Amsterdam, 1986.

Andreas Defant:

Fachbereich Mathematik

Universitaet

D-26111 Oldenburg

Germany

E-mail address: defant@mathematik.uni-oldenburg.de

Domingo García:

Departamento de Análisis Matemático

Universidad de Valencia

Doctor Moliner 50

46100 Burjasot (Valencia)

Spain

E-mail address: domingo.garcia@uv.es

Manuel Maestre:

Departamento de Análisis Matemático

Universidad de Valencia

Doctor Moliner 50

46100 Burjasot (Valencia)

Spain

E-mail address: manuel.maestre@uv.es

Primera versió rebuda el 26 de maig de 2009, darrera versió rebuda el 21 de juliol de 2009. 